\title{
UNIVERSAL BINDING ENERGY RELATIONS FOR BIMETALLIC INTERFACES AND RELATED SYSTEMS
}

\author{
J.R. Smith, J. Ferrante ${ }^{+}$and J.H. Rose ${ }^{++}$ \\ Physics Department, General Motors Research Laboratories, \\ Warren, MI 48090-9055, U.S.A \\ 'National Aeronautios and Space Administration, Lewis Research Center, \\ Cleveland, Ohio 44135, U.S.A. \\ ${ }^{++}$Ames Laboratory, U.S. Dept. of Energy, Iowa State University, \\ Ames, Iowa 50011, U.S.A.
}

\begin{abstract}
Résumé - Nous avons calculé la structure électronfque autocohérente et i'énergie totale en fonction de la distance entre les surfaces de 10 contacts bimétalliques - toutes combinaisons de $\mathrm{Al}(111), \mathrm{Mg}(0001), \mathrm{Zn}(0001)$ et $\mathrm{Na}(110)$. On a trouvé que les effets "volume" étaient très importants sur 1 'interaction bimétaliłque. L'énergie cinétique amorce 1'adhésion, alors que 1'énergie échange-corrélation est le facteur dominant quant à la force de 1 'adhésion. I1 a été trouvé qu'une formule unique entre l'énergie totale et la distance interfaciale décrit avec préciston l'énergétique de tous les dix contacts, ainst que plusieurs cas de chemisorption, cohésion et interaction moléculaire.
\end{abstract}

\begin{abstract}
We have computed the self-consistent electronic structure and total energy as a function of the distance between surfaces of ten bimetallic contacts -- all combinations of $\mathrm{Al}(111), \mathrm{Mg}(0001), \mathrm{Zn}(0001)$, and $\mathrm{Na}(110)$. "Volume" effects are found to be very important to the bimetallic interaction. The kinetic energy initiates the bond, while the exchangecorrelation energy is the dominant contributor to the strength of the adhesive bond. It was found that a single relation between the total energy and interfacial distance accurately describes the energetics of all ten contacts, as well as many cases of chemisorption, cohesion, and molecular interaction.
\end{abstract}

Metals in intimate contact interact strongly, leading to significant changes in interfacial electronlc structure as a function of separation between the surfaces. These strong adhesive bonds can play an important role /1/ in the deposition of metal films, grain boundary energetics, friction and wear, and fracture.

We have reported earlier /2/ on self-consistent electronic calculations of total energies as a function of interfacial spacing for cases in which the two metals are identical. Here we report the results of the first fully self-consistent calculations of adhesive energies and electronic structure for bimetallic interfaces. A11 combinations of $\mathrm{Al}(111), \mathrm{Zn}(0001), \mathrm{Mg}(0001)$, and $\mathrm{Na}(110)$ are treated. "Volume" dependent terms were included self-consistently (the pair potential approximation was not made). The results for adhesive energetics led to the discovery /3/ of universality in binding energy relations, a universality which extends to chemisorption $/ 4 /$, cohesion, and diatomic-molecule energetics $|5-7|$. 
The model used in our bimetallic calculations is deptcted in F1g. 1 for an Al-Mg contact.

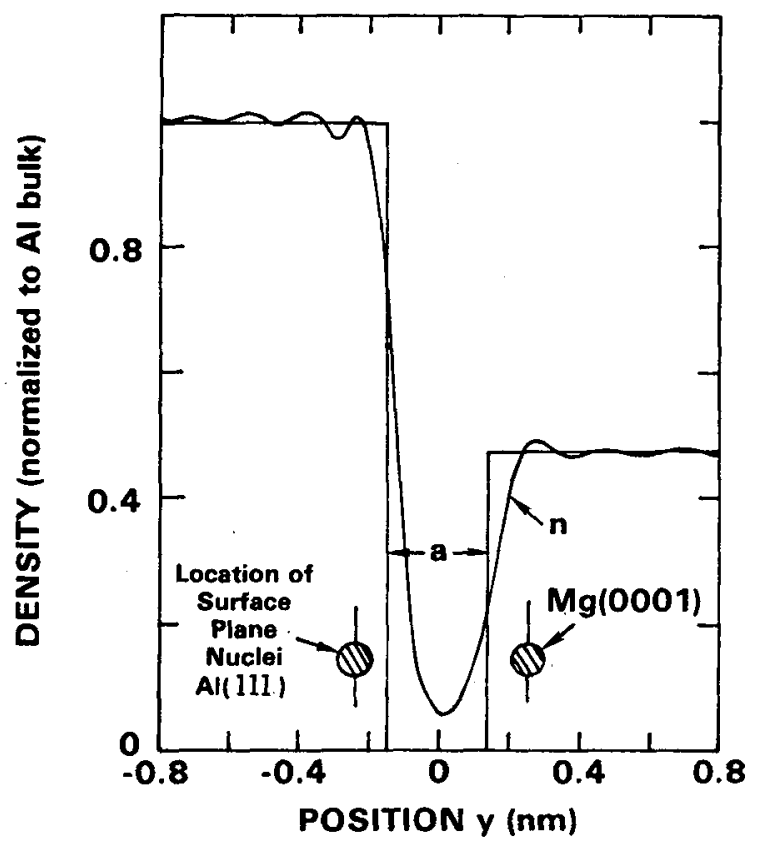

Fig. 1 Electron number density $n$ and jellium ion charge density $n_{+}$for an $\mathrm{Al}-\mathrm{Mg}$ contact. When $\mathrm{a}=0.0$, the distance between $\mathrm{Mg}$ and $\mathrm{Al}$ atomic planes is $\left(d_{A 1}+d_{M g}\right) / 2$, where $d_{A 1}$ and $d_{M g}$ are the respective bulk interplanar spacings.

The electron number density is determined in zeroth-order by solving the Kohn-Sham equations /8/ self-consistently for two jellium slabs separated by the distance a as shown. The effect of crystalline arrays of pseudopotentials for each of the two metals is then introduced via first-order perturbation theory. For detalls of the method see Refs. /2/ and /9/.

Some effects of the self-consistent charge rearrangement are shown in Fig. 2 for the example of an $\mathrm{A} 1-\mathrm{Na}$ contact for $\mathrm{a}=0$.

The upper panel, Fig. (2a), is a plot of the self-consistent electron density distribution minus the overlapped, solid-vacuum electron densities for Al and $\mathrm{Na}$. It shows that there is a nontrivial charge rearrangement due to the interaction between the two metals. It also suggests that there is an electron transfer from the $\mathrm{Na}$ (the metal on the right) to the Al (the metal on the left). This is consistent with the work function of the $\mathrm{Na}$ being smaller than that of Al. Fig. (2b) is a plot of the self-consistent electron potential minus the noninteracting or solid-vacuum potentlals. Note the charge transfer leads to a contact potential $\Delta \mathrm{V}$ of $0.81 \mathrm{eV}$. However, the potential actually peaks over $4 \mathrm{eV}$ higher than the contact potential. Thus the commonly used contact potential really does not adequately describe what takes place in the interface. 

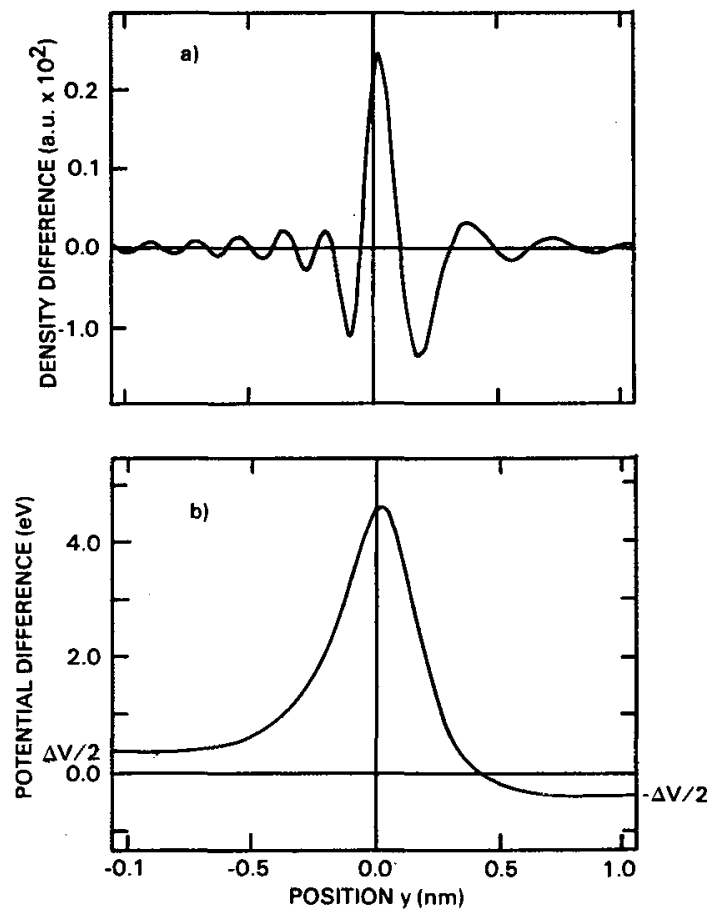

Fig. 2 a) Self-consistent electron densities, $n(y, a)$, minus overlapped-solidvacuum electron densities for $a=0.0$ in an $\mathrm{A} 1-\mathrm{Na}$ contact, (A1 on the left). This density difference plot shows the charge rearrangement due to interaction between the Al and Na surfaces.

b) Self-consistent electron potentials $\nu_{\text {eff }}(n ; y)$, minus overlapped-solidvacuum electron potentials, for $a=0.0$ in an Al-Na contact.

The adhesive energy is defined as

$$
E_{a d}(a)=[E(a)-E(\infty)] / 2 A \text {, }
$$

where $E$ is the total energy and $A$ is the interfacial cross-sectional area. For identical metals, $E_{a d}$ is the negative of the surface energy when a is at the energy minimum. GIven the self-consistent electron density distribution, one can employ density functional theory to obtain $\mathrm{E}(\mathrm{a})$ (see Refs. 2 and 9 for details). In Fig. 3 we show a breakdown of the components of the total energy as a function of separation for an $\mathrm{Al}-\mathrm{Mg}$ contact. Note that the kinetic energy initiates the bond. In analogy with the molecular bond $/ 10 /$, smoothing of the wave functions in the bonding region lowers the kinetic energy with respect to infinite separation. The electrostatic energy is repulsive at large separations, but becones attractive at smaller separations. The dominant binding term or "glue" is the exchange-correlation energy, as is evident from Fig. 3. The dominant repulsive term is the kinetic energy at small separation. It should be pointed out that the 
kinetic and exchange-correlation energies are "volume" terms. These volume terms, which are not adequately treated in the usual pair-potential approaches, are the dominant repulsive and attractive energies.

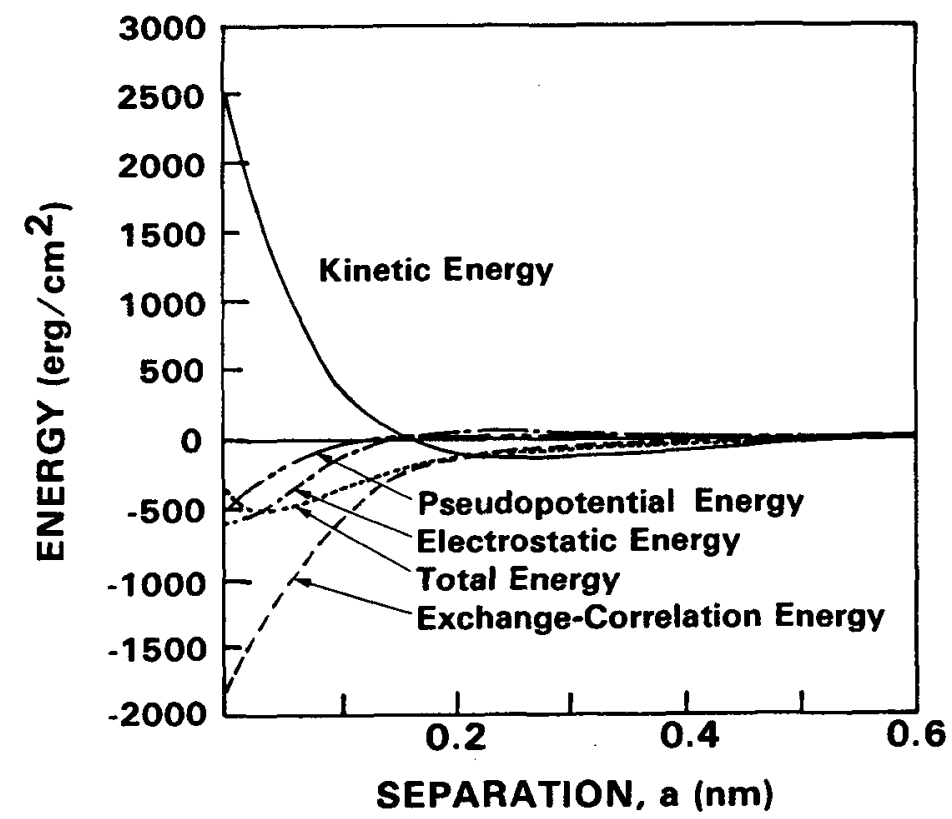

Fig. 3 Self-consistent energy components of the binding energy for an $\mathrm{A} 1(111)-\mathrm{Mg}(0001)$ contact .

The total energy has a minimum near $a=0$, and the range of the strong bonding is about $0.2 \mathrm{~nm}$. This is typical of the ten bimetallic contacts we treated. We wish now to look further for systematics in the adhesive energy relations.

There are quite a variety of shapes and amplitudes in plots of total energy versus separation for the ten bimetallic contacts we considered /3/. We have found /5/, however, that these ten curves can be scaled quite accurately into a single, universal curve as shown In Fig. 4. The deviation of the separation a from the equllibrium value $a_{m}$ is scaled as

$$
a^{*} \equiv\left(a-a_{m}\right) / l
$$

The adhesive energy is scaled as

$$
\mathrm{E}_{\mathrm{ad}}^{*}(\mathrm{a}) \equiv \mathrm{E}_{\mathrm{ad}}(\mathrm{a}) / \Delta \mathrm{E} \text {, }
$$

where

$$
\Delta E=E_{a d}\left(a_{m}\right)
$$


and

$$
\ell \equiv\left\{\Delta \mathrm{E}\left[\frac{\mathrm{d}^{2} \mathrm{E}_{\mathrm{ad}}(\mathrm{a})}{\mathrm{da}^{2}}\right]_{\mathrm{a}_{\mathrm{m}}}\right\}^{-1}
$$

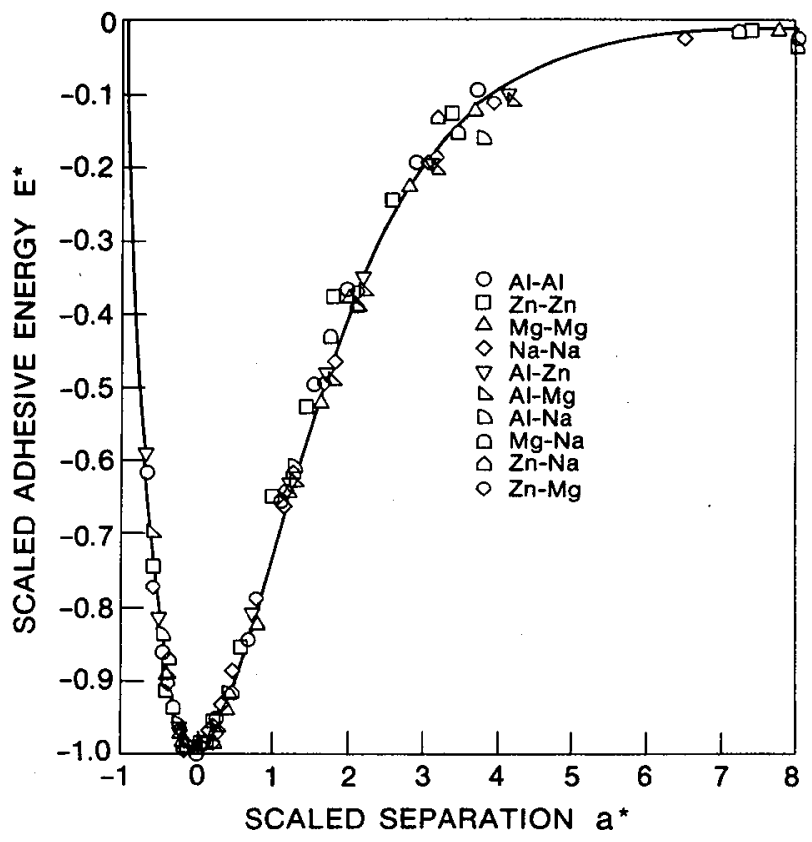

Fig. 4 Adhesive energy results scaled as described in the text (see Eqs. 2-5).

\begin{abstract}
A untversal curve ensues even though the bulk densities of the metals vary by a factor of eight.

This universality is not limited to interfaces between simple metals. Figure 5 contains curves for a molecule $\left(\mathrm{H}_{2}^{+}\right)$, bulk metal (Mo), chemisorbed atom (0), and bimetallic interface $(\mathrm{Al}-\mathrm{Z} \mathrm{n})$. We commented earlier on how Fig. 3 showed a similarity between the bimetallic bond and the molecular bond. Now we can see that there can in some cases be a detailed correspondence between the molecular and the metallic bond, in terms of the shape of the binding energy curve. This correspondence has some generality, as discussed in Ref. 5. There is an underlying simplicity in nature which has not been recognized heretofore.
\end{abstract}




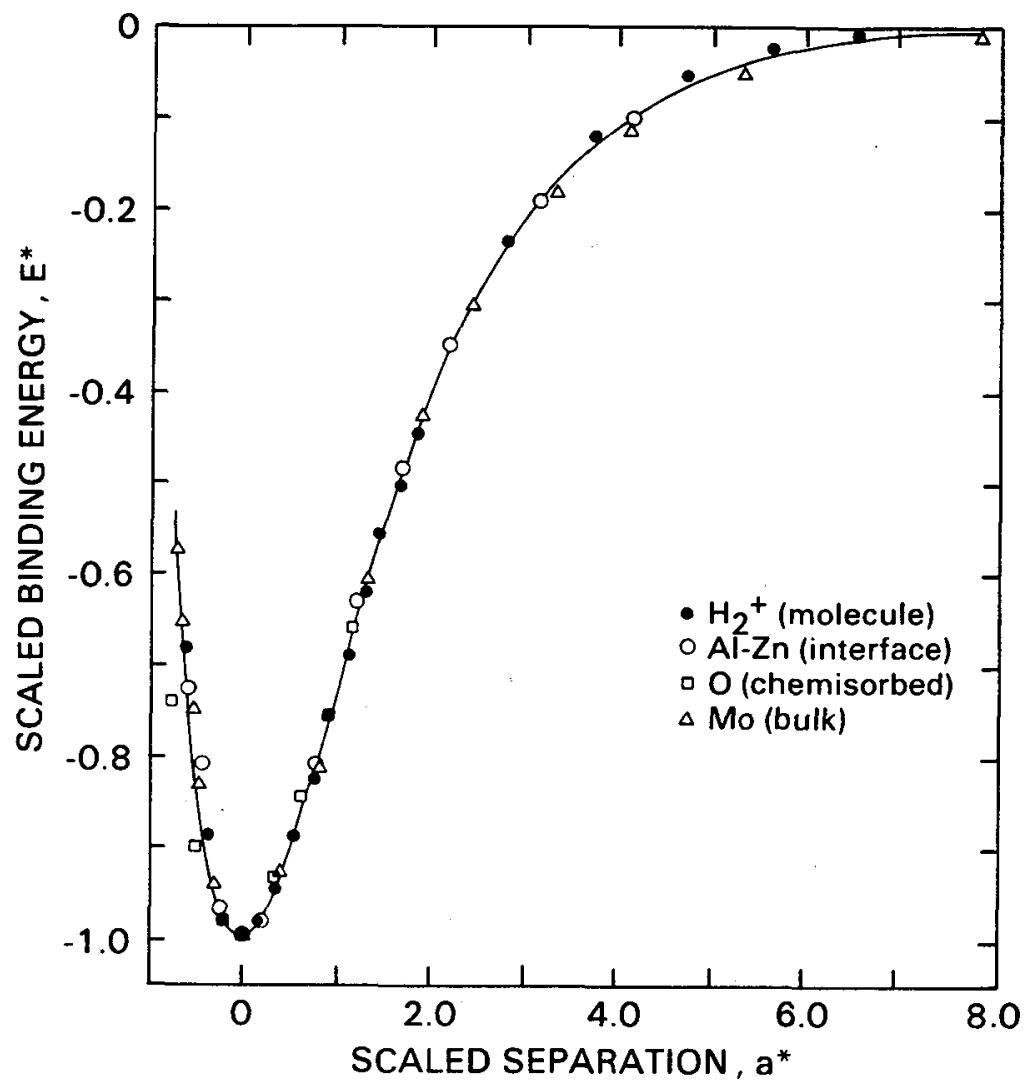

Fig. 5 Binding energy as a function of interatomic separation for four systems as noted, scaled as described in the text. The $\mathrm{H}_{2}^{+}$results were taken from Ref. 11, the A1-Zn interface energies from Ref. 9, the oxygen chemisorption binding energy from Ref. 12, and the Mo binding energy from Ref. 13.

\section{REFERENCES}

1. D. H. Buckley, J. Colloid and Interface Sc1. 58, 36 (1977).

2. John Ferrante and John R. Smith, Phys. Rev, B 19, 3911 (1979).

3. J. H. Rose, John Ferrante, and John R. Smith, Phys. Rev. Letters 47, 675 (1981).

4. John R. Smith, John Ferrante, and J. H. Rose, Phys. Rev. B 25, 1419 (1982);

John P. Perdew and John R. Smith, Surface Sci. 41, L295 (1984).

5. John Ferrante, John R. Smith, and James H. Rose, Phys. Rev. Letters 50, 1385 (1983); Phys. Rev. B 28, 1835 (1983). 
6. John R. Smith, James H. Rose, John Ferrante, and Francisco Guinea in "ManyBody Phenomena at Surfaces," edited D. Langreth and H. Suhl, Academic Press, 1984 , p. 159.

7. Francisco Guinea, James H. Rose, John R. Smith, and John Ferrante, Appl. Phys. Lett. 44,53 (1984).

8. W. Kohn and L. J. Sham, Phys. Rev. 140, A 1133 (1965).

9. John Ferrante and John R. Smith, Phys. Rev. B (to be published).

10. M. J. Feinberg and Klaus Ruendenberg, J. Chem. Phys. 54, 1495 (1971). C. Woodrow Wilson, Jr., and William A. Goddard, III., Theoret. Chem. Acta (Ber1.) 26, 195 (1972).

11. J. C. Slater, "Quantum Theory of Molecules and Solids," (McGraw-Hill, New York, 1963), Vol. 1.

12. J. K. Norskov and N. D. Lang, Phys. Rev. B 21, 2131 (1980).

13. A. E. Carlsson, C. D. Gelatt, and H. Ehrenreich, Philos. Mag. A 41, 241 (1980). 